Pacific Journal of Mathematics

AN INEQUALITY FOR THE HILBERT TRANSFORM 


\section{AN INEQUALITY FOR THE HILBERT TRANSFORM}

\section{RAY REDHEFFER}

The purpose of this paper is to give a general inequality for the Hilbert transform that clearly distinguishes conditions of global and local integrability. The former conditions are associated with a certain product $[f, g]_{m}$, the latter with another product $\{f, g\}_{p}$. The resulting statement contains, as corollaries, a number of inequalities for the Hilbert transform that have not been hitherto noted. Presentation of these is a second objective here. It turns out that the general theorem also includes, in sharpened form, several classical inequalities of Hardy and Littlewood, Babenko, and others. Proof of these sharpened forms is a third objective.

By means of the theory of Calderón and Zygmund results similar to those of this paper can be established for Hilbert transforms in $n$ dimensions and for singular integrals of more general types. However, this is not done here.

1. Definitions and notation. We use $f, g, h$ and so on for complex-valued functions of a real variable, $u$ or $x$, on the domain

$$
(-\infty, 0) \cup(0, \infty) \text {. }
$$

All functions are assumed locally integrable, that is, integrable in the sense of Lebesgue over each compact subinterval of the above domain, and $p$ and $q$ are complementary Lebesgue exponents. Thus,

$$
p \geqq 1, \quad \frac{1}{p}+\frac{1}{q}=1 .
$$

The statement $f \in L^{p}$ means that $f$ is locally integrable and $|f|^{p}$ is integrable on $(-\infty, \infty)$, the omitted point 0 being irrelevant here. Otherwise, our integrals are interpreted whenever possible as Cauchy principal values near $0, x$ and $\pm \infty$. This applies, in particular, to the Hilbert transform

$$
\widehat{f}(x)=\int_{-\infty}^{\infty} \frac{f(u)}{x-u} d u
$$

and to the modified Hilbert transform $\hat{f}_{m}$ introduced below .

We define, as usual, $L=L^{1}$, and

$$
\|f\|_{p}=\left(\int_{-\infty}^{\infty}|f(x)|^{p} d x\right)^{1 / p}, \quad p \geqq 1 .
$$

Any inequality of form $P \leqq Q$ is considered to be vacuously fulfilled 
if $Q=\infty$, and $\|f\|_{p}=\infty$ if $f$ is not in $L^{p}$.

A well-known theorem of Riesz asserts that

$$
\|\hat{f}\|_{p} \leqq R_{p}\|f\|_{p}, \quad p>1
$$

where $R_{p}$ is constant. The smallest constant that can appear in this inequality is called the Riesz constant; for example, $R_{2}=\pi$. Since the Riesz theorem fails for $p=1$ we assume once for all that the functions $f$ and $g$ belong locally to $L^{p}$ for some $p>1$. However they need not belong to $L^{p}$ on $(-\infty, \infty)$ for any $p$. (The same effect can be achieved by declaring that $R_{p}=\infty$ for $p=1$, which we do, but. perhaps the hypothesis mentioned here is clearer).

Throughout this paper $a, b, c, A, B, C, \alpha, \beta, \rho, \sigma$ denote real constants, which may be different in different contexts, and $\left\{x_{n}\right\}$ denotes a real sequence satisfying

$$
x_{n}>0, \lambda \leqq \frac{x_{n+1}}{x_{n}} \leqq \mu, \quad-\infty<n<\infty
$$

where $\lambda$ and $\mu$ are constants such that $1<\lambda \leqq \mu$. It is said, briefly, that $\left\{x_{n}\right\}$ belongs to $(\lambda, \mu)$. The values $\lambda, \mu$ and the sequence $\left\{x_{n}\right\}$ are regarded as fixed and are not always carried as explicit parameters.

We could introduce another sequence $\left\{y_{n}\right\}$ such that $\left\{-y_{n}\right\}$ belongs to $(\lambda, \mu)$ and thus obtain a more general formulation of the results below. Instead, we refer both $f(x)$ and $f(-x)$ to the same sequence $\left\{x_{n}\right\}$ for $x>0$, placing a higher priority upon simplicity of statement than upon maximum generality.

To avoid overburdening these introductory remarks, other definitions are presented as needed. The modified Hilbert transform $\hat{f}_{m}$, which forms the subject of this paper, is introduced in the next section, together with an error term $[f]_{m}$. A functional $J_{p}(\varphi)$ that plays a role in the formulation of new inequalities is introduced in $\S 3$, and the products $\{f, g\}_{p}$ and $[f, g]_{m}$ are introduced in $\S 4$, to permit the statement of Theorem 1. These expressions all have the meanings assigned to them on their first introduction, even when mentioned later in the paper.

2. A modified Hilbert transform. The Hilbert transform exists only for a narrow class of functions. We propose to enlarge this class by allowing $f(u)$ a behavior at $-\infty, u_{0}$, and $\infty$ that might make the integral diverge. Clearly $u_{0}=0$ involves no loss of generality and is assumed henceforward.

Near $u=0$ and $|u|=\infty$ we have, respectively, 


$$
\begin{aligned}
& \frac{1}{x-u}=\frac{1}{x}\left[1+\frac{u}{x}+\left(\frac{u}{x}\right)^{2}+\cdots\right], \\
& \frac{1}{x-u}=-\frac{1}{x}\left[\frac{x}{u}+\left(\frac{x}{u}\right)^{2}+\cdots\right],
\end{aligned}
$$

which give suitable approximations when terminated. Without loss of generality the same terminating exponent, $m-1$, is taken for both series. For $m \geqq 1$, then, let

$$
\begin{array}{ll}
K_{m}(r)=1+r+r^{2}+\cdots+r^{m-1}, & |r| \leqq 1, \\
K_{m}(r)+K_{m}\left(\frac{1}{r}\right)=1 & |r|>1 .
\end{array}
$$

The modified Hilbert transform, which allows singular behavior of $f$ at 0 and at $\pm \infty$, is defined by

$$
\hat{f}_{m}(x)=\int_{-\infty}^{\infty}\left[\frac{1}{x-u}-\frac{1}{x} K_{m}\left(\frac{u}{x}\right)\right] f(u) d u, \quad m=1,2,3, \cdots
$$

Near 0 and $\pm \infty$ the integral behaves like

$$
\int_{|u|<\varepsilon} u^{m} f(u)[1+O(u)] d u, \quad \int_{|u|>1 / \varepsilon} \frac{f(u)}{u^{m}}\left[1+O\left(\frac{1}{u}\right)\right] d u
$$

respectively, hence exists for a larger class $\{f\}$ than that available for $\hat{f}$. Later we shall require absolute convergence of the integrals above. It is nevertheless appropriate to consider $\hat{f}_{m}$ as a Cauchy principal value, because the earlier terms of the series then exist for a broader class of functions $f$ than they otherwise would.

The structure of the formula for $\hat{f}_{m}$ is somewhat clearer when $\hat{f}$ exists. In that case

$$
\hat{f}_{1}(x)=\hat{f}(x)-\bar{f}(x), \quad \text { where } \quad \bar{f}(x)=\frac{1}{x} \int_{-|x|}^{|x|} f(u) d u .
$$

This reduces to $\hat{f}$ when $f$ is odd. For $m \geqq 2$

$$
\begin{aligned}
\hat{f}_{m}(x)= & \hat{f}_{1}(x)-\frac{1}{x} \int_{|u|<|x|}\left[\left(\frac{u}{x}\right)+\left(\frac{u}{x}\right)^{2}+\cdots+\left(\frac{u}{x}\right)^{m-1}\right] f(u) d u \\
& +\frac{1}{x} \int_{|u| \geqq|x|}\left[\left(\frac{x}{u}\right)+\left(\frac{x}{u}\right)^{2}+\cdots+\left(\frac{x}{u}\right)^{m-1}\right] f(u) d u .
\end{aligned}
$$

Hence, about half the terms in $\hat{f}_{m}-\hat{f}$ can be dropped when $f$ is even, and about half can be dropped when $f$ is odd.

Equation (2) gives $\hat{f}_{m+1}(x)-\hat{f}_{m}(x)$ as an integral with respect to $u$. Another form is obtained by writing $-u$ for $u$, and still a third is obtained by averaging these. The third expression indicates that 


$$
\left|\hat{f}_{m+1}(x)-\hat{f}_{m}(x)\right| \leqq\left.\frac{1}{|x|}\right|_{-\infty} ^{\infty} \min \left(\left|\frac{x}{u}\right|^{m},\left|-\frac{u}{x}\right|^{m}\right) \frac{\left|f(u)+(-)^{m} f(-u)\right|}{2} d u
$$

Thus, $\hat{f}_{m+1}-\hat{f}_{m}$ depends only on the even part of $f$ if $m$ is even, and only on the odd part if $m$ is odd. For any function $f$ we define

$$
[f(x)]_{m}=\int_{-\infty}^{\infty} \min \left(\left|\frac{x}{u}\right|^{m},\left|-\frac{u}{x}\right|^{m}\right)|f(u)| d u .
$$

Then $\left|\hat{f}_{m+1}(x)-\hat{f}_{m}(x)\right| \leqq[f(x)]_{m} /|x|$, and a sharper result can be obtained by distinguishing even and odd parts. The expression $[f]_{m}$ is often encountered in the sequel.

To illustrate the generality of $\hat{f}_{m}$, it may be mentioned that $\hat{f}_{m}(x)$ exists for $m=1,2,3 \cdots$ when $f(x)=e^{x} \sin e^{2 x}$, though $|f(x)|$ behaves substantially like $e^{x}$ in this case. The theory of this paper is not applicable, since $[f(x)]_{m}=\infty$ for all $m$. However the choice $f(x)=$ $(1+|x|)^{a} \sin e^{x}$ again ensures existence of $\hat{f}_{m}$ for all $m$, and here $[f]_{m}<\infty$ for $m>a+1$. It is possible to have singularities at 0 as well as at $\infty$; for instance, if $f(x)=x^{a}$ for $x>0, f(x)=0$ elsewhere, then $\hat{f}_{m}$ exists for $|a+1|<m$, while $\hat{f}$ does exist when $a \leqq-1$. A different generalization of $\hat{f}(x)$, originating in an idea of Achiezer, has been considered by Koizumi [16].

We shall find it expedient to choose $m$ large enough to make the various integrals in our analysis converge, and then deduce results for smaller $m$ by direct examination of $[f(x)]_{k}, k<m$. The behavior of $\bar{f}(x)$ and of $[f(x)]_{k}$ is so simple that we regard these functions as "known" in the same sense that $f$ is known. A theorem about $\hat{f}_{m}$ is, in essence, a theorem also about $\hat{f}$.

Comparison of $\hat{f}$ and $\hat{f}_{m}$ is facilitated by a theorem of Hardy, Littlewood, and Pólya [12] which reads as follows: Let

$$
G(x)=\int_{0}^{x} g(t) d t, \quad r>1 ; \quad G(x)=\int_{x}^{\infty} g(t) d t, \quad r<1,
$$

where $g \geqq 0$. Then for $p>1$,

$$
\int_{0}^{\infty} x^{-r} G^{p} d x \leqq\left(\frac{p}{|r-1|}\right)^{p} \int_{0}^{\infty} x^{p-r} g^{p} d x
$$

Furthermore, the constant on the right is sharp.

For $r>1$ this applies to the part of $[f]_{m}$ for which the integration is over $|u|<|x|$, and for $r<1$ it applies to the other part. By a short calculation we get the following: Let $\alpha=a-1 / q$ satisfy $|\alpha|<m$. Then

$$
\left\|x^{a-1}[f(x)]_{m}\right\|_{p} \leqq \frac{4}{m-|\alpha|}\left\|x^{a} f\right\|_{p} .
$$


It is also true that

$$
\left\|x^{a} \bar{f}(x)\right\|_{p} \leqq \frac{2}{\left|\alpha^{\prime}\right|}\left\|x^{a} f(x)\right\|_{p}
$$

provided $\alpha<0$. These results can be applied with $f$ replaced by its even or odd part, as explained above.

3. A survey of results obtained here. The result of this paper which is most closely connected with well-known theorems is Corollary 6. This asserts that

$$
\left\|x^{a} \hat{f}_{m}\right\|_{p} \leqq C\left(R_{p}+\frac{m}{m-|\alpha|}\right)\left\|x^{a} f\right\|_{p}, \quad a^{\prime}=a-\frac{1}{q},
$$

where $|\alpha|<m$ and where $C$ is an absolute constant. In 1936 Hardy and Littlewood [10] proved for $p>1$ that

$$
\left\|x^{a} f\right\|_{p}<\infty \text { implies }\left\|x^{a} \hat{f}\right\|_{p}<\infty
$$

provided $f$ is even and $-2<\alpha<0$. In 1944 this was refined (for the circle) by $K$. Chen [4], who showed that

$$
\left\|x^{a} \hat{f}\right\|_{p} \leqq C(a, p)\left\|x^{a} f\right\|_{p}
$$

for some constant $C(a, p),-2<\alpha<0$. Both follow from (7) with $m=2$. Indeed, since $f$ is even, $\hat{f}_{2}=\hat{f}_{1}$, and hence (7) applies to $\hat{f}_{1}$ with $|\alpha|<2$. In the Hardy-Littlewood case $\alpha<0$ and (6) together with the above gives

$$
\left\|x^{a} \hat{f}\right\|_{p} \leqq C\left(R_{p}-\frac{1}{\alpha(2+\alpha)}\right)\left\|x^{a} f\right\|_{p}, \quad-2<\alpha<0,
$$

where $C$ is an absolute constant.

In 1948 Babenko [1] obtained Chen's result without assuming $f$ even, provided $-1<\alpha<0$. This follows from (7) with $m=1$, and in the more explicit form

$$
\left\|x^{a} \hat{f}\right\|_{p} \leqq C\left(R_{p}-\frac{1}{\alpha(1+\alpha)}\right)\left\|x^{a} f\right\|_{p}, \quad-1<\alpha<0,
$$

where $C$ is an absolute constant.

In 1958 Flett [8] remarked that it ought to be possible to get Babenko's theorem for a larger range of $\alpha$ when $f$ is odd, as had already been done by Hardy-Littlewood and Chen for $f$ even. Flett showed, in fact, that the result holds for $|\alpha|<1$, on $(-\pi, \pi)$, provided $f$ is odd, periodic and integrable. A sharpened form of Flett's theorem on $(-\infty, \infty)$ follows from (7) with $m=1$.

Since the integration in (7) involves the region $|x|<1$ as well 
as $|x|>1$, the effect of changing the parameter $a$ is by no means obvious at first glance. Corollary 7 gives a more general version of (7), in which $x^{a}$ on the left is replaced by two different powers, one for $|x|<1$ and one for $|x|>1$. Similarly, $x^{a}$ on the right is replaced by two different powers. The resulting inequality reduces to (7) again in case all exponents are equal.

For example, the choice $\alpha=\beta=0, \rho=\sigma=-\alpha$ in Corollary 7 gives the following: Let $a$ satisfy

$$
\left|a+\frac{1}{q}\right| \leqq m-\delta
$$

where $\delta$ is a positive constant. Then there exists a constant $C_{m}$, depending on $m$ alone, such that

$$
\left\|\frac{\hat{f}_{m}}{(1+|x|)^{a}}\right\|_{p} \leqq C_{m}\left(R_{p}+\frac{1}{\delta}\right)\left\|\frac{f}{(1+|x|)^{a}}\right\|_{p} .
$$

The choice $m=1, a=\alpha / p$, where $0 \leqq \alpha<1$, gives a sharpened form of a theorem of Koizumi [15]. The case $a=1$ is also worthy of note, since we can then take $1 / \delta=p$ for $m \geqq 2$. In particular, $m=p=2$ gives

$$
\int_{-\infty}^{\infty} \frac{\left|\hat{f}_{2}(x)\right|^{2}}{1+x^{2}} d x \leqq C \int_{-\infty}^{\infty} \frac{|f(x)|^{2}}{1+x^{2}} d x
$$

where $C$ is an absolute constant. Thus the transform taking $f$ into $\hat{f}_{2}$ belongs to the Wiener class.

Corollary 7 shows a remarkable symmetry in the conditions for $|x|<1$ and $|x|>1$. The weight $(1+|x|)^{-a}$ considered above behaves like 1 near 0 , and like $|x|^{-a}$ near $\infty$. We could just as well construct a weight that behaves like 1 near $\infty$ and like $|x|^{-x}$ near 0 . Indeed, if the weight $(1+|x|)^{-a}$ is replaced by

$$
\left(1+\frac{1}{|x|}\right)^{a}
$$

then the same inequality holds, and with the same condition on $a$. This follows by taking $\rho=\sigma=0, \alpha=\beta=-a$ in Corollary 7 .

It should perhaps be mentioned that these results can be combined with the Marcinkiewicz-Zygmund interpolation theorem [20] to get results of the form

$$
\int_{-\infty}^{\infty} \varphi\left(\hat{f}_{m}\right) w(x) d x \leqq C_{\varphi} \int_{-\infty}^{\infty} \varphi(f) W(x) d x
$$

for suitable functions $\varphi$. Since the technique is well known [6], [14], [15], the details are not repeated here. Our main objectives lie in a somewhat different direction, as described next. 
Results of the foregoing types fail when $p=1$. For instance, if $f \in L$ then it does not follow that $\hat{f} \in L$, or even that $\hat{f}_{m} \in L$ for any $m$. However, if $f$ admits a majorant $F \in L$ such that $x^{\rho} F(x)$ is monotone for some $\rho$, for $x>0$, and also admits such a majorant for $x<0$, then $\hat{f}_{1} \in L$ does follow. This is the content of Corollary 2 . Corollary 2 was in part suggested by, and strongly generalizes, an unpublished result of Matzayev on even entire functions [19].

The assumption of a monotone majorant is very restrictive. For example, a function as simple as $|\log | \cos x||$ is excluded, through for many purposes this function behaves like a constant. Here we introduce majorants that are in a certain sense of regular growth, and yet have infinities. This is accomplished by use of the functional

$$
J_{p}(\varphi)=\sup _{-\infty<n<\infty}\left(\frac{1}{x_{n+1}-x_{n}} \int_{x_{n}}^{x_{n+1}}|\varphi(x)|^{p} d x\right)^{1 / p}
$$

where $\left\{x_{n}\right\}$ is the sequence belonging to $(\lambda, \mu)$ mentioned in $\S 1$. The majorants have the general form $|f| \leqq F \varphi$, where $J_{p}(\varphi) \leqq 1$ and where $F$ has the desired regularity. It can be thought that $F$ is a smooth carrier of the singular mass distribution $\varphi$.

Every function $f$ which is locally $L^{p}$ can be written in the form $f=F \varnothing$ for $x>0$, where $F$ is constant on each interval $\left(x_{n}, x_{n+1}\right)$ and where $J_{p}(\varphi) \leqq 1$. To see this, let

$$
I_{n}=\left(\frac{1}{x_{n+1}-x_{n}} \int_{x_{n}}^{x_{n+1}}|f(x)|^{p} d x\right)^{1 / p}
$$

so that $J_{p}(f)=\sup I_{n}$. If $I_{n}>0$ we define

$$
F(x)=I_{n}, \quad \varphi(x)=\frac{f(x)}{I_{n}}, \quad x_{n}<x<x_{n+1}
$$

while if $I_{n}=0$, the definition $F=0, \varphi=1$ is used. Then $f=F \varnothing$ almost everywhere, and $J_{p}(\varphi)=1$. We set $F(x)=0$ for $x<0$.

The particular decomposition mentioned above makes $F \geqq 0$ and minimizes $|F|$ subject to the constraints: $J_{p}(\varphi) \leqq 1, F=$ const on $\left(x_{n}, x_{n+1}\right)$. This is called the minimal decomposition.

For brevity, it is said that $G(x)$ belongs to $a$ if $x^{-a} G(x)$ decreases and $x^{a} G(x)$ increases for $x>0$. Corollary 8 then reads as follows: Let $|f(x)|+|f(-x)|$ have the minimal decomposition $F(x) \varphi(x)$ for $x>0$. Let $|g(x)|+|g(-x)|=G(x) \psi(x)$ where $G(x)$ belongs to some constant $a<m$ and where $J_{q}(\psi) \leqq 1$. Then there exists a constant $C$, independent of $f$ and $g$, such that

$$
\left\|\hat{f}_{m} g\right\|_{1} \leqq C\|F G\|_{1} \text {. }
$$

The form of $C$ is determined explicitly. In particular, if 


$$
\lambda \leqq 1+\frac{1}{m}, \quad \mu \leqq \lambda^{2}
$$

as can be assumed with only slight loss of generality, then

$$
C=C_{0}\left(R_{p}+\frac{1}{m(m-a)}\right)
$$

where $C_{0}$ is an absolute constant.

Other corollaries have various monotony properties for $F$, in the majorant $|f| \leqq F \varphi$, and greater flexibility in the weight $g,|g| \leqq G \psi$. The general significance of these results is that the $L$ behavior of $\hat{f}_{m}$ relative to the weight $g$ is largely determined by the $L$ behavior of $F G$ in these decompositions, and that more regularity in one of the functions $F, G$ allows less in the other.

A class of functions that is well adapted to majorants of the above types is the class of periodic functions. If $|f(x)|$ has period $\omega>0$ let $x_{n}=\omega 2^{n}$, so that $\lambda=\mu=2$. Then for $n \geqq 0$

$$
I_{n}=\left(\frac{1}{\omega} \int_{0}^{\omega}|f(x)|^{p} d x\right)^{1 / p}
$$

and the function $F$ in the decomposition $f=F \varphi$ is constant. Corollary 10 indicates that

$$
\left\|\widehat{f}_{m} g\right\|_{1} \leqq C\|g\|_{1}\left(\int_{0}^{\omega}|f(x)|^{p}\right)^{1 / p}, \quad m \geqq 2,
$$

where $g$ is any weight such that $g=0$ for $x<\omega$, and $|g(x)| x^{\rho}$ is monotone for some $\rho$. The dependence of $C$ on the relevant parameters is also given, and the actual statement is much more general. These results apply to broad classes of functions $f(x)=P[Q(x)]$ where $P$ is periodic and $Q$ is sufficiently well behaved. For instance $f(x)=$ $P(\log x)$ makes $f(\lambda x)=f(x)$ for $\lambda=e^{\omega}$, and the choice $x_{n}=\lambda^{n}$ gives ${ }^{2} I_{n}=$ const. again.

4. The main theorem. In this section we state the main theorem, from which the other results follow as corollaries. The formulation requires two products, $[f, g]_{m}$ and $\{f, g\}_{p}$. The first of these correlates $f$ on the whole range $(-\infty, \infty)$ with $g$ on the whole range $(-\infty, \infty)$ and is called the global product. The second correlates $f$ and $g$ only on overlapping intervals $\left(x_{n-1}, x_{n+1}\right)$ and is called the local product.

The global product, $[f, g]_{m}$, is the integral of $[f]_{m}|g|$ or $|f|[g]_{m}$. Thus,

$$
[f, g]_{m}=\int_{-\infty}^{\infty} \int_{-\infty}^{\infty} \min \left(\left|\frac{x}{u}\right|^{m},\left|\frac{u}{x}\right|^{m}\right)|f(u)||g(x)| d u d x
$$


(We integrate with respect to $x$ from $-\infty$ to $\infty$ so that $[f, g]_{m}$ will be symmetric. In most applications $g(x)=0$ for $x<0$.) Existence of $[f]_{m}$ in (4) is equivalent to convergence of

$$
\int_{|u|<1}|u|^{m}|f(u)| d u \text { and } \int_{|u|>1}|u|^{-m}|f(u)| d u .
$$

Hence, the larger $m$ is, the larger is the class of functions for which $[f, g]_{m}<\infty$.

The local product $\{f, g\}_{p}$ is defined by

$$
\{f, g\}_{p}=\sum_{n=-\infty}^{n=\infty}\left(\int_{x_{n-1}}^{x_{n+1}}|f(x)|^{p} d x\right)^{1 / p}\left(\int_{x_{n-1}}^{x_{n+1}}|g(x)|^{q} d x\right)^{1 / q}
$$

and $\{f, g\}_{p}=\infty$ if the series diverges or a term is infinite. We could avoid the dependence on $\left\{x_{n}\right\}$ by taking the inf over $\left\{x_{n}\right\}$ belonging to $(\lambda, \mu)$, but that would not always be convenient.

Thd main theorem of this paper is:

Theorem 1. Let $A_{p}=2 R_{p}$, where $R_{p}$ is the Riesz constant, and let

$$
B_{m}=\max \left[\frac{\lambda}{\lambda-1}, \frac{\mu^{2}}{\mu^{2}-1}\left(\mu^{2 m}-1\right)\right] .
$$

Let $g(x)=0$ for $x<0$. Then for all $m, p, \lambda$ and $\mu$

$$
\left\|x \widehat{f}_{m}(x) g(x)\right\|_{1} \leqq A_{p}\{f, x g\}_{p}+B_{m}[f, g]_{m}{ }^{\prime} .
$$

The conclusion could also be written in the form

$$
\left\|\hat{f}_{m} g\right\|_{1} \leqq A_{p}\{f, g\}_{p}+B_{m}\left[f, \frac{g}{x}\right]_{m} .
$$

The earlier statement is preferred because it allows the roles of $f$ and $g$ to be interchanged, by virtue of

$$
[f, g]_{m}=[g, f]_{m}, \quad\{f, g\}_{p}=\{g, f\}_{q} .
$$

It turns out that the factor $x$ can be transferred from $g$ to $f$ in $\{f, x g\}_{p}$, with only trivial modification, while simple examples show that

$$
[f, x g]_{m}<\infty, \quad[x f, g]_{m}=\infty
$$

are compatible for every value of $m$. The symmetry is of some interest because one often has a different hypothesis for $f$ from that for $g$. The fact that $x \hat{f}_{m} g$ and $x f \hat{g}_{m}$ satisfy substantially the same inequality is, then, not quite obvious.

The factor $x$, which can be transferred from $g$ to $f$ in $\{f, g\}_{p}$ but not in $[f, g]_{m}$, is responsible for some subtleties in the following 
analysis, as is the fact that $\{f, g\}_{p}$ involves overlapping rather than disjoint intervals in the sum.

5. Proof of the main theorem. If $R$ and $S$ are positive functions of $x$ let

$$
f^{*}(x ; R, S)=\int_{x-R}^{x+S} \frac{f(u)}{x-u} d u
$$

for $x>0$, so that

$$
x f^{*}(x ; R, S)=\int_{x-R}^{x+S} \frac{1}{1-\left(\frac{u}{x}\right)} f(u) d u, \quad x>0 .
$$

By (2), with $K=K_{m}$ for short,

$$
x \hat{f}_{m}(x)=\int_{-\infty}^{\infty}\left[\frac{1}{1-\left(\frac{u}{x}\right)}-K\left(\frac{u}{x}\right)\right] f(u) d u .
$$

For $|r|<1$, equation (1) gives

$$
K(r)=\frac{1-r^{m}}{1-r}, \frac{1}{1-r}-K(r)=\frac{r^{m}}{1-r}, \quad(|r|<1) .
$$

Since $K(r)=1-K(1 / r)$ for $|r|>1$ we have also

$$
K(r)=\frac{1-r^{1-m}}{1-r}, \frac{1}{1-r}-K(r)=\frac{r^{1-m}}{1-r}, \quad(|r|>1) .
$$

Applying this with $r=u / x$ gives

$$
x \hat{f}_{m}(x)-x f^{*}(x ; R, S)=\int_{-\infty}^{\infty} k\left(\frac{u}{x}\right) f(u) d u
$$

where $k(r)$ has the values

$$
\frac{r^{1-m}}{1-r}, \frac{r^{m}}{1-r}, \frac{1-r^{m}}{r-1}, \frac{1-r^{1-m}}{r-1}, \frac{r^{1-m}}{1-r}
$$

on the respective intervals

$$
(-\infty,-x), \quad(-x, x-R), \quad(x-R, x), \quad(x, x+S), \quad(x+S, \infty) .
$$

The intervals of the variable $r=u / x$ corresponding to these intervals of the integration variable $u$ are respectively

$$
(-\infty,-1),\left(-1, \frac{x-R}{x}\right),\left(\frac{x-R}{x}, 1\right),\left(1, \frac{x+S}{x}\right),\left(\frac{x+S}{x}, \infty\right) .
$$

To introduce $[f, g]_{m}$ we need a factor $|u / x|^{m}$ on intervals where 
$|u|<x$, and a factor $|x / u|^{m}$ on intervals where $|u|>x$. Hence we set $\rho=1 / r$ and write $k(r)$ in the form

$$
\rho^{m} \frac{r}{1-r}, \quad r^{m} \frac{1}{1-r}, \quad-r^{m} \frac{1-\rho^{m}}{1-\rho} \rho, \quad \rho^{m} \frac{r^{m-1}-1}{r-1} r, \quad \rho^{m} \frac{r}{1-r}
$$

on the above intervals, respectively. In each case the factor multiplying $r^{m}$ or $\rho^{m}$ is monotone, as seen when this factor is written as a geometric series. Hence, the maximum of this factor occurs at an end point of the corresponding interval and can be found by inspection. To estimate these factors we need values for $R$ and $S$, given next.

Let $\left\{x_{n}\right\}$ be a sequence belonging to $(\lambda, \mu)$ and such that $\{f, x g\}_{p}$ converges; if there is no such sequence then there is nothing to prove. We define $R$ and $S$ by

$$
R=x-x_{n-1}, \quad S=x_{n+2}-x \quad\left(x_{n} \leqq x \leqq x_{n+1}\right)
$$

and observe that $(x-R) / x=x_{n-1} / x,(x+S) / x=x_{n+2} / x$. Hence,

$$
\frac{1}{\mu^{2}} \leqq \frac{x-R}{x} \leqq \frac{1}{\lambda}, \quad \lambda \leqq \frac{x+S}{x} \leqq \mu^{2} .
$$

These results can be used to estimate the coefficients of $r^{m}$ or $\rho^{m}$ in the above formulas for $k(r)$. The result is that

$$
\left|k\left(\frac{u}{x}\right)\right| \leqq B_{m} \min \left(\left|\frac{u}{x}\right|^{m},\left|\frac{x}{u}\right|^{m}\right)
$$

for $x>0$, where $B_{m}$ is the constant of the theorem. Namely, this holds on $x_{n} \leqq x<x_{n+1}$ for every $n$ and hence it holds on $(0, \infty)$. From (9) it follows that

$$
\left|x \widehat{f}_{m}(x)-x f^{*}(x ; R, S)\right| \leqq B_{m}[f(x)]_{m}
$$

where $[f(x)]_{m}$ is given by (4). If this is multiplied by $|g(x)|$ and integrated from 0 to $\infty$ the right-hand side is $B_{m}[f, g]_{m}$, since we have assumed $g(x)=0$ for $x<0$.

To get a similar estimate for the integral of $\left|x f^{*}(x ; R, S) g(x)\right|$ note that

$$
\begin{aligned}
& \int_{x_{n}}^{x_{n+1}} x\left|f^{*}(x ; R, S) g(x)\right| d x=\int_{x_{n}}^{x_{n+1}}\left|\int_{x_{n-1}}^{x_{n+2}} \frac{f(u)}{x-u} d u\right||x g(x)| d x \\
& \quad \leqq R_{p}\left(\int_{x_{n-1}}^{x_{n+2}}|f(x)|^{p} d x\right)^{1 / p}\left(\int_{x_{n}}^{x_{n+1}}|x g(x)|^{q} d x\right)^{1 / q}
\end{aligned}
$$

where in the last step we used the Schwarz-Hölder inequality and the Riesz theorem for Hilbert transforms of class $L^{p}$. It is well 
known that

$$
\frac{1}{2}\left(a^{1 / p}+b^{1 / p}\right) \leqq(a+b)^{1 / p} \leqq a^{1 / p}+b^{1 / p} \quad(a \geqq 0, b \geqq 0, p \geqq 1)
$$

and hence

$$
\left(\int_{x_{n-1}}^{x_{n+2}}|f(x)|^{p} d x\right)^{1 / p} \leqq\left(\int_{x_{n-1}}^{x_{n+1}}|f(x)|^{p}\right)^{1 / p}+\left(\int_{x_{n}}^{x_{n+2}}|f(x)|^{p}\right)^{1 / p} .
$$

This shows that the coefficient of $R_{p}$ above is dominated by the sum of two adjacent terms in the series defining $\{f, x g\}_{p}$. Upon multiplying the inequality

$$
\left|x \widehat{f}_{m}(x)\right| \leqq x\left|\hat{f}_{m}(x)-f^{*}(x ; R, S)\right|+x\left|f^{*}(x ; R, S)\right|
$$

by $|g(x)|$, integrating from $x_{n}$ to $x_{n+1}$, and summing on $n$, we get the inequality of the theorem.

6. Convergence theorems. Here we give some easy consequences of Theorem 1 that do not involve detailed estimates. Corollaries 1-5 use Theorem 1 only for $m \leqq 2$, and in several cases only for $p=1 / 2$. Proof of Theorem 1 for this case is somewhat simpler, but not enough simpler to be worth writing out again. For the reader's convenience we recall (3), namely, $\hat{f}_{1}=\hat{f}-\bar{f}$, if $\hat{f}$ exists, where $\hat{f}$ is the Hilbert transform and $\bar{f}$ is twice the average of $f(u)$ sign $x$ on $(-|x|,|x|)$. In this section the letter $S$ denotes the set of sequences $\left\{x_{n}\right\}$ satisfying

$$
x_{n}>0, \quad \text { inf } \frac{x_{n+1}}{x_{n}}>1, \quad \text { sup } \frac{x_{n+1}}{x_{n}}<\infty .
$$

It is well known that the Riesz theorem fails for $p=1$, so that $f \in L$ does not imply $\hat{f} \in L$. However we shall establish the following:

Corollary 1. For $x>0$, suppose $|f(x)|$ admits a majorant $F(x) \in L$ which is constant on each interval $\left(x_{n}, x_{n+1}\right)$ of some sequence $\left\{x_{n}\right\} \in S$. Suppose $|f(-x)|$ also admits such a majorant. Then $\hat{f}_{1} \in L$.

For proof let $g(x)=1 /|x|$ for $|x|>1$ and $g(x)=1$ for $|x|<1$. Then $[g(x)]_{1}$ as given by (4) with $m=1$ is bounded, and hence $f \in L$ implies $[g, f]_{1}<\infty$. This gives $[f, g]_{1}<\infty$, since $[f, g]_{m}=[g, f]_{m}$. Also we have the following approximate relations for $p=2, x_{n}>0$ :

$$
\begin{aligned}
\{f, x g\}_{p} & \doteq \sum\left(\left|x_{n}\right| \int_{x_{n-1}}^{x_{n+1}}|f(x)|^{2} d x\right)^{1 / 2} \\
& \leqq \sum\left|x_{n}\right|\left\{\left[F\left(x_{n-1}^{\prime}\right)\right]^{2}+\left[F\left(x_{n}^{\prime}\right)\right]^{2}\right\}^{1 / 2} \\
& \leqq \sum\left|x_{n}\right|\left[F\left(x_{n-1}^{\prime}\right)+F\left(x_{n}^{\prime}\right)\right]
\end{aligned}
$$


Here $x_{n}^{\prime}$ is any point on $\left(x_{n}, x_{n+1}\right)$. Since $F \in L$ the latter series converges and Corollary 1 follows.

CoRollary 2. Suppose $|f|$ admits a majorant $F \in L$ such that $x^{\rho} F(x)$ is monotone for some constant $\rho$ as $x \rightarrow \infty$, and also such that $|x|^{\circ} F(x)$ is monotone for some constant $\rho$ as $x \rightarrow-\infty$. Then $\hat{f} \in L$.

For proof let $x>0$ and, to fix ideas, let $x^{\rho} F(x)$ be increasing. Taking $x_{n}=2^{n}$ we have

$$
2^{-\rho} F\left(x_{n}\right) \leqq F(x) \leqq 2^{\rho} F\left(x_{n+1}\right) \quad\left(x_{n} \leqq x \leqq x_{n+1}\right) .
$$

The left-hand relation together, with $F \in L$ gives

$$
\sum_{1}^{\infty} x_{n} F\left(x_{n}\right)<\infty
$$

and the right-hand relation then gives a majorant of the type needed in Corollary 1. Discussion of the case in which $x^{\circ} F(x)$ is decreasing and of the case $x<0$ is similar.

The choice $f(u)=u^{-1}(\log u)^{-2}$ for $u \geqq 2$ shows that $\hat{f}_{1}$ cannot be replaced by $\hat{f}$ in Corollaries 1 and 2 . The same example applies to several results given below.

CoROLlaRy 3. Let $|f(u)| \leqq F(|u|)$ and $|g(u)| \leqq G(|u|)$ where $F(x)$ and $G(x)$ are 0 for $x<0$. Suppose $x F G \in L$, and suppose $x^{\rho} F(x)$ and $x^{\rho} G(x)$ are increasing for some constant $\rho<2$. Then $x \widehat{f}_{1} g \in L$.

A calculation similar to that in Corollary 1 gives $\{f, x g\}_{p}<\infty$ even if it is not assumed that $\rho<2$. The integral for $[f, g]_{1}$ can be found by integrating over the region $R$ where $|u|<|x|$ and over the complementary region $R^{\prime}$. The first integral does not exceed

$$
\int_{R}|u|^{\rho} F(|u|)|u|^{-\rho} \frac{|u|}{|x|} G(|x|) d u d x \leqq \frac{4}{2-\rho} \int_{0}^{\infty} x F(x) G(x) d x
$$

and hence is finite. By symmetry the same inequality holds for the integral over $R^{\prime}$ and Corollary 3 follows.

The choice $f(u)=u^{-2}, g(u)=(\log u)^{-2}$ for $u \geqq 2$ shows that the condition $\rho<2$ cannot be replaced by $\rho \leqq 2$ in Corollary 3. Namely, Theorem 1 gives $x \hat{f}_{2} g \in L$ under these conditions, but $x\left(\hat{f}_{1}-\hat{f}_{2}\right) g$ is not in $L$.

When $\rho<1$ in Corollary 3 then $\hat{f}_{1}$ can be replaced by $\hat{f}$, through such replacement is not permitted when $\rho \geqq 1$. This is so because if

$$
|f(u)+f(-u)| \leqq F(|u|)
$$


where $x^{\rho} F(x)$ is increasing for some constant $\rho<1$, then

$$
|\bar{f}(x)| \leqq \frac{F(|x|)}{1-\rho} .
$$

It was observed above that in $\{f, g\}_{p}$ the two functions $f$ and $g$ are compared only locally. We can allow $f$ to be very large on a given interval $\left(x_{n}, x_{n+1}\right)$ if $g$ is correspondingly small on that interval. The following example is an extreme case:

Corollary 4. Let $\left\{x_{n}\right\}=X$ be a given sequence belonging to $S$. Suppose the support of $f$ is contained in a set $X_{f}$ of closed intervals

$$
x_{n} \leqq x \leqq x_{n+1}
$$

of $X$, and suppose the support of $g$ is contained in another such set, $X_{g}$, disjoint from $X_{f}$. If there is a $p>1$ such that $f \in L^{p}$ and $g \in L^{q}$ locally, then $[f, g]_{m}<\infty \Rightarrow x \hat{f}_{m} g \in L$.

The proof is obvious, because $\{f, g x\}_{p}=0$ under the stated conditions.

CoRollary 5. Let $\varphi(u)$ have period $\omega>0$ and let $\varphi \in L^{p}$ locally for some $p>1$. Define

$$
F_{a}(x)=\int_{\omega}^{\infty} \frac{x u^{a}}{x^{2}-u^{2}} \varphi(u) d u-\delta_{a} \frac{\log x}{x} \frac{1}{\omega} \int_{0}^{\omega} \varphi(u) d u,
$$

where $\delta_{a}=1$ for $a=-1$ and $\delta_{a}=0$ otherwise. Then

$$
\int_{\omega}^{\infty} x^{a} w(x) d x<\infty \Rightarrow \int_{\omega}^{\infty}\left|F_{a}(x)\right| w(x) d x<\infty, \quad-1 \leqq a<1,
$$

where $w(x)$ is any nonnegative weight such that $x^{\circ} w(x)$ is monotone for some $\rho$.

We apply Theorem 1 with $f(u)=|u|^{a} \varphi(|u|)$ for $|u| \geqq \omega$, otherwise $f(u)=0$. Also $g(x)=w(x) / x$ for $x \geqq \omega$, otherwise $g(x)=0$. If $x_{n}$ is large then, approximately,

$$
\left(\int_{x_{n-1}}^{x_{n+1}}|\varphi(u)|^{p} d u\right)^{1 / p} \doteq \frac{x_{n}}{\omega}\left(\int_{0}^{\omega}|\varphi(u)|^{p} d u\right)^{1 / p}=c x_{n}
$$

where $c$ is constant. Thus, for purposes of convergence,

$$
\{f, x g\}_{p}=\left\{x^{a} \varphi, w\right\}_{p} \doteq\left\{\varphi, x^{a} w\right\}_{p} \doteq\left\{c, x^{a} w\right\}_{p} .
$$

Since $\left(x^{a} w\right) x^{\rho}$ is monotone for some $\rho$, the hypothesis $x^{a} w \in L$ makes $\{f, x g\}_{p}<\infty$ as in the proof of Corollary 1 or 2 . 
The behavior of $[f, g]_{m}$ is not satisfactory when $m=1$ and so we pick $m=2$. Computation of $[f(x)]_{2}$ leads to consideration of

$$
\frac{1}{x^{2}} \int_{\omega}^{x} u^{a+2}|\varphi(u)| d u+x^{2} \int_{x}^{\infty} u^{a-2}|\varphi(u)| d u .
$$

Since $\varphi \in L$ on its period it is easily checked that the above expression has the order of magnitude $x^{a+1}$ as $x \rightarrow \infty$, just as it would if $\varphi$ were constant. Hence $g[f]_{2}$ has the order of magnitude $w x^{a}$ and therefore $[f, g]_{2}<\infty$.

Theorem 1 now gives $w \hat{f}_{2} \in L$. Since $f$ is even, $f_{2}=\hat{f}-\bar{f}$. The left-hand integral in $F_{a}(x)$ is $\hat{f}(x) / 2$ and the right-hand expression is $\bar{f}(x) / 2$, apart from a term of order $x^{a}$. This is seen when we write $\varphi=(\varphi-M)+M$, where $M$ is the mean value of $\varphi$ over a period. The term $\varphi-M$ has a bounded integral, and integration by parts gives the desired conclusion.

Corollary 5 shows, for example, that the two functions

$$
f(u)=\frac{\sin u}{u}, \quad f(u)=\left|\frac{\sin u}{u}\right|
$$

behave differently with respect to convergence. If $w(x)=(\log x)^{-2}$ for $x \geqq 2 \pi$ then the first function satisfies $\hat{f} w \in L$ and the second does not.

7. Properties of the global product. Here we collect some properties of $[f, g]_{m}$ that are useful for sharpening and generalizing the previous corollaries, as explained in $\S 3$.

Property 1. Let $a+b=1$ and let $\alpha=a-1 / q=1 / p-b$ satisfy $|\alpha|<m$. Then

$$
[f, g]_{m} \leqq \frac{8 m}{m^{2}-\alpha^{2}}\left\|x^{a} f\right\|_{p}\left\|x^{b} g\right\|_{q} .
$$

Suppose first that $f=g=0$ for $x<0$. In this case

where

$$
[f, g]_{m}=\int_{0}^{\infty} \int_{0}^{\infty} k(x, y) x^{a}|f(x)| y^{b}|g(y)| d x d y
$$

$$
k(x, y)=\frac{1}{x^{a}} \frac{1}{y^{b}} \min \left[\left(\frac{x}{y}\right)^{m},\left(\frac{y}{x}\right)^{m}\right] .
$$

Since $k(x, y)$ is homogeneous of degree -1 we can apply Hardy's generalization of Hilbert's inequality [11]. By a short computation

$$
\int_{0}^{\infty} k(x, 1) x^{-1 / p} d x=\int_{0}^{\infty} k(1, y) y^{-1 / q} d y=\frac{2 m}{m^{2}-\alpha^{2}} .
$$


The Hardy-Hilbert inequality now gives

$$
[f, g]_{m} \leqq C\left\|x^{a} f\right\|_{p}\left\|x^{b} g\right\|_{q}
$$

where $C=2 m /\left(m^{2}-\alpha^{2}\right)$, and shows that this result is sharp.

To get the general case, note that the arbitrary functions $f$ and: $g$ can be written

$$
f=f_{1}+f_{2}, \quad g=g_{1}+g_{2}
$$

where $f_{1}=g_{1}=0$ for $x<0$ and $f_{2}=g_{2}=0$ for $x>0$. By symmetry (12) applies to each pair $f_{i}, g_{j}$, that is, it applies in each quadrant. Therefore

$$
\begin{aligned}
{[f, g]_{m} } & \leqq \sum\left[f_{i}, g_{j}\right]_{m} \leqq C \sum\left\|x^{a} f_{i}\right\|_{p}\left\|x^{b} g_{j}\right\|_{q} \\
& =C\left(\sum\left\|x^{a} f_{i}\right\|_{p}\right)\left(\sum\left\|x^{b} g_{j}\right\|_{q}\right) \leqq 4 C\left\|x^{a} f\right\|_{p}\left\|x^{b} g\right\|_{q},
\end{aligned}
$$

where in the last step we used the inequality on the left in (10). This completes the proof.

Property 2. Let $a, b, A, B$ be constants such that

$$
\begin{array}{lll}
a \leqq \frac{1}{q}+m-\delta, & b \leqq \frac{1}{p}+m-\delta, & a+b \leqq 1 \\
A \geqq \frac{1}{q}-m+\delta, & B \geqq \frac{1}{p}-m+\delta, & A+B \geqq 1
\end{array}
$$

where $0<\delta \leqq m$. Let

Then

$$
\begin{aligned}
& r(x)=|x|^{a}, \quad s(x)=|x|^{b} \quad(|x| \leqq 1) \\
& r(x)=|x|^{A}, \quad s(x)=|x|^{B} \quad(|x| \geqq 1) .
\end{aligned}
$$

$$
[f, g]_{m} \leqq \frac{32}{\delta}\|r f\|_{p}\|s g\|_{q} .
$$

We give the proof first for the case in which $a+b=1, A+B=1$. In this case

$$
\left|a-\frac{1}{q}\right|=\left|b-\frac{1}{p}\right| \leqq m-\delta
$$

and likewise for $A$ and $B$. Let

$$
f(x)=f_{1}(x)+f_{2}(x), \quad g(x)=g_{1}(x)+g_{2}(x)
$$

where $f_{1}=g_{1}=0$ for $|x| \geqq 1$ and $f_{2}=g_{2}=0$ for $|x|<1$. Property 1 gives

$$
\left[f_{1}, g_{1}\right]_{m} \leqq \frac{8}{\delta}\left\|x^{a} f_{1}\right\|_{p}\left\|x^{b} g_{1}\right\|_{q},
$$

and similarly for $\left[f_{2}, g_{2}\right]_{m}$ with $A$ and $B$ on the right. 
To estimate $\left[f_{2}, g_{1}\right]_{m}$ consider the integral

$$
\int_{1}^{\infty} x^{-m-A} x^{A} f_{2}(x)\left(\int_{0}^{1} u^{m-b} u^{b} g_{1}(u) d u\right) d x .
$$

By two uses of the Schwarz-Hölder inequalily it is found that

$$
\left[f_{2}, g_{1}\right]_{m} \leqq \frac{2}{\delta}\left(p^{-1 / p}\right)\left(q^{-1 / q}\right)\left\|x^{A} f_{2}\right\|_{p}\left\|x^{b} g_{1}\right\|_{q} .
$$

(The factor 2 arises from the extension to the other quadrants). A similar result holds for $\left[f_{1}, g_{2}\right]_{m}$ with $a$ and $B$ on the right. Since $p^{-1 / p} \leqq 1$ these results can be combined as in the proof of Property 1 . This gives the desired conclusion, under the additional condition that $a+b=A+B=1$.

To extend the result, let $R_{\delta}$ be the region of the $(a, b)$ plane in which the inequality of Property 2 holds for fixed $\delta$. Then if $\left(a_{0}, b_{0}\right) \in R_{\delta}$ it is easily seen that $(a, b) \in R_{\delta}$ whenever $a<a_{0}$ and $b<b_{0}$. Similar remarks apply to $(A, B)$. This completes the proof of Property 2 , as can be seen by a sketch.

If, besides the hypothesis of Property $2, a, b, A, B$ satisfy the additional condition

$$
a+b \leqq 1-\delta, \quad A+B \geqq 1+\delta
$$

it is possible to deduce the conclusion by use of the Schwarz-Hölder inequality, without using Property 1 and without the assumption $\delta \leqq m$. In fact, the following generalization holds in this case: Let $r$ and $s$ be even functions such that $r(x) x^{-a}$ and $s(x) x^{-b}$ are decreasing on $(0,1)$, while $r(x) x^{-A}$ and $s(x) x^{-B}$ are increasing on $(1, \infty)$. Let $a, b, A, B$ satisfy the hypothesis of Property 2 and let the condition $\delta \leqq m$ be replaced by (13). Then

$$
[f, g]_{m} \leqq \frac{32}{\delta r(1) s(1)}\|r f\|_{p}\|s g\|_{q} .
$$

In particular, this applies if $\delta \geqq 2 m$, since (13) holds automatically in that case. Thus Property 2 holds for $\delta \geqq 2 m$ as well as for $0 \leqq \delta \leqq m$. If $m \leqq \delta \leqq 2 m$ then Property 2 holds with the constant $m$ on the right instead of $\delta$.

Property 3. Let $f(x)>0$ and $g(x)>0$ for $x>0$ and let $f(x)=$ $g(x)=0$ for $x<0$. Given $a<m$, consider the four functions

$$
x^{1+a} f(x), \quad x^{1+a} g(x), \quad x^{1-a} f(x), \quad x^{1-a} g(x) .
$$

Suppose one of the following conditions holds:

(i) The first and second functions both increase; 
Then

(ii) the first increases, the third decreases;

(iii) the second increases, the fourth decreases;

(iv) the third and fourth decrease.

$$
[f, g]_{m} \leqq \frac{2}{m-a}\|x f g\|_{1} .
$$

The proof follows by using the two possible orders of integration in the region $u<x$, and the two possible orders in the region $u>x$; we omit the routine details.

As the reader will recall, the functions used in the ZygmundMarcinkiewicz interpolation theorem satisfy inequalities of the form

$$
\int_{u}^{\infty} \frac{\varphi(t)}{t^{b+1}} d t=O\left(\frac{\varphi(u)}{u^{b}}\right), \quad \int_{1}^{u} \frac{\varphi(t)}{t^{a+1}} d t=O\left(\frac{\varphi(u)}{u^{a}}\right)
$$

and similar inequalities on $(u, 1)$ and $(0, u)$; cf. [6], [14], for example. The monotony conditions of Property 3 could be replaced by more general conditions of this kind. In particular, in place of cases (ii) and (iii) one could just assume

$$
[f(x)]_{m} \leqq \alpha|x f(x)| \text { or }[g(x)]_{m}<\alpha|x g(x)|
$$

where $\alpha$ is constant, and

$$
[f, g]_{m} \leqq \alpha\|x f g\|_{1}
$$

then follows by inspection.

The reader preferring these more general conditions will find that the following proofs are virtually unchanged. The monotony formulation of Property 3 is used here because it is simpler.

Let $r(x)>0$ for $x>0$ and $r(x)=0$ for $x<0$. It is said that $r$ is right-majorized with constant $\alpha$ if

$$
r(x) \leqq \alpha r(t x), \quad 1 \leqq t \leqq \mu,
$$

and that $r$ is left majorized with constant $\alpha$ if the above holds with $1 / \mu \leqq t \leqq 1$.

Property 4. Let $r=0$ for $x<0, r>0$ for $x>0$, and let $C$ have the value

$$
C=\frac{\alpha^{2}}{\mu} \frac{\mu-1}{\lambda-1}, \quad C=\alpha \beta, \quad C=\beta^{2} \lambda \frac{\mu-1}{\lambda-1}
$$

according as $r$ is right-majorized with constant $\alpha$, both right and left majorized with constants $\alpha, \beta$, or left majorized with constant B. Then

$$
[r \varphi, g]_{m} \leqq C \mu^{2 m}[r, g]_{m} J_{1}(\varphi)
$$


For proof we compare $[r \varphi]_{m}$ with $[r]_{m}$. Given $x$, let

$$
L(u)=L_{x}(u)=\min \left(\left|\frac{x}{u}\right|,\left|\frac{u}{x}\right|\right), \quad u>0 .
$$

By distinguishing the three cases in which the interval $(u / \mu, u \mu)$ is wholly at the left of $|x|$, or wholly at the right, or contains $|x|$, it is seen that $L(u)$ is both right and left majorized with the constant $\mu$, no matter what value $x$ may have. Thus the function

$$
R(u)=R_{x}(u)=r(u)[L(u)]^{m}
$$

is right-majorized with constant $\alpha \mu^{m}$ if $r$ is right-majorized with constant $\alpha$. In that case

$$
[r \varphi]_{m}=\Sigma \int_{x_{n}}^{x_{n+1}} R(u)|\varphi(u)| d u \leqq \alpha \mu^{m} R\left(x_{n+1}\right) d_{n+1} J_{1}(\varphi)
$$

where $d_{n}=x_{n}-x_{n-1}$. On the other hand

$$
[r(x)]_{m}=\sum \int_{x_{n+1}}^{x_{n+2}} R(u) d u \geqq\left(\mu^{m} \alpha\right)^{-1} R\left(x_{n+1}\right) d_{n+2} .
$$

Without loss of generality it can be assumed that $J_{1}(\varphi)=1$. Under this condition we want to choose a constant $C$ so that $C d_{n+2} \geqq\left(\mu^{m} \alpha\right)^{2} d_{n+1}$. Since

$$
\frac{d_{n+1}}{d_{n+2}}=\frac{x_{n+1}-x_{n}}{x_{n+2}-x_{n+1}} \leqq \frac{1-1 / \mu}{\lambda-1}
$$

we get $C$ as the first constant in Property 4. With this $C$,

$$
[f, g]_{m}=\int_{-\infty}^{\infty}[r \varphi]_{m}|g| d x \leqq C \int_{0}^{\infty}[r]_{m}|g| d x=C[r, g]_{m} .
$$

Proof of the other two statements is similar.

8. Properties of the local product. The results of this section pertain to the product $\{f, g\}_{p}$. We assume throughout that all functions are 0 for $x<0$. By the Schwarz-Hölder inequalities for integrals and for series,

$$
2\|f g\|_{1} \leqq\{f, g\}_{p} \leqq 2\|f\|_{p}\|g\|_{q} \cdot
$$

We wish to get conditions under which the first inequality can be effectively reversed, so that $\{f, g\}_{p} \leqq C\|f g\|_{1}$ for some constant $C$.

Property 5. Suppose $r$ and $s$ are both right majorized, or are both left majorized, with the respective constants $\alpha$ for $r$ and $\beta$ for $s$. Suppose also $r s=1$. Then 


$$
(\alpha \beta)^{-2}\{f, g\}_{p} \leqq\{r f, s g\}_{p} \leqq(\alpha \beta)^{2}\{f, g\}_{p} .
$$

If $r$ and $s$ are right-majorized then

$$
r(x) \leqq \alpha^{2} r\left(x_{n+1}\right), \quad s(x) \leqq \beta^{2} s\left(x_{n+1}\right)
$$

for $x_{n+1} \leqq x \leqq x_{n+1}$ and the desired result follows by inspection of the general term in the series for $\{r f, s g\}_{p}$. The case in which they are left-majorized is similar.

Property 6. Let $r$ be right-majorized with constant $\alpha$ and leftmajorized with constant $\beta$. Then

$$
\{r \varphi, g\}_{p} \leqq \alpha \beta\{r, g\}_{p} J_{p}(\varphi) .
$$

This follows by comparison of the two integrals

$$
\int_{x_{n-1}}^{x_{n+1}}|r \varphi|^{p} d x, \quad \int_{x_{n-1}}^{x_{n+1}} r^{p} d x
$$

as in the proof of Property 4. Since a more difficult case is considered later the proof is omitted.

If both $r$ and $s$ satisfy the hypothesis, one can use Property 6 twice and get

$$
\{r \varphi, s \psi\}_{p} \leqq(\alpha \beta)^{2}\{r, s\}_{p} J_{p}(\varphi) J_{q}(\psi)
$$

We shall obtain a similar result when $r$ and $s$ satisfy only one-sided conditions.

Property 7. Suppose $r$ and $s$ are both right majorized, or are both left majorized, with the respective constants $\alpha$ for $r$ and $\beta$ for s. Then

where

$$
\{r \varphi, s \psi\}_{p} \leqq C\{r, s\}_{p}, \quad\{r \varphi, s \psi\}_{p} \leqq \alpha \beta C\|r s\|_{1}
$$

$$
C=4(\alpha \beta)^{2} \lambda \mu \frac{\mu-1}{\lambda-1} J_{p}(\varphi) J_{q}(\psi) .
$$

For proof let $d_{n}=x_{n}-x_{n-1}$ and suppose $(r, s)$ are right-majorized. Then (10) gives

$$
\left(\int_{x_{n-1}}^{x_{n+1}}|r \varphi|^{p} d x\right)^{1 / p} \leqq \alpha\left[d_{n+1}^{1 / p} r\left(x_{n+1}\right)+d_{n}^{1 / p} r\left(x_{n}\right)\right] J_{p}(\varphi) .
$$

We replace $d_{n}$ and $d_{n+1}$ by $\max \left(d_{n}, d_{n+1}\right)$, and, without loss of generality, we take $J_{p}(\varphi)=J_{q}(\psi)=1$. The above together with the analogous relation for $s \psi$ shows that the general term in $\{r \psi, s \psi\}_{p}$ does not exceed 


$$
\alpha \beta \max \left(d_{n}, d_{n+1}\right)\left[r\left(x_{n+1}\right)+r\left(x_{n}\right)\right]\left[s\left(x_{n+1}\right)+s\left(x_{n}\right)\right] .
$$

On the other hand (10) also gives

$$
\left(\int_{x_{n}}^{x_{n+2}} r^{p} d x\right)^{1 / p} \geqq \frac{1}{2 \alpha}\left[d_{n+2}^{1 / p} r\left(x_{n+1}\right)+d_{n+1}^{1 / p} r\left(x_{n}\right)\right]
$$

and hence the general term in $C\{r, s\}_{p}$ is at least

$$
\frac{C}{4 \alpha \beta} \min \left(d_{n+2}, d_{n+1}\right)\left[r\left(x_{n+1}\right)+r\left(x_{n}\right)\right]\left[s\left(x_{n+1}\right)+s\left(x_{n}\right)\right] .
$$

It suffices, therefore, to choose $C$ so that

$$
C>4(\alpha \beta)^{2} \frac{\max \left(d_{n}, d_{n+1}\right)}{\min \left(d_{n+2}, d_{n+1}\right)} .
$$

Since

$$
\left(1-\frac{1}{\lambda}\right) x_{n} \leqq d_{n} \leqq\left(1-\frac{1}{\mu}\right) x_{n}, \quad(\lambda-1) x_{n} \leqq d_{n+1} \leqq(\mu-1) x_{n}
$$

the numerator is at most $(\mu-1) x_{n}$ and the denominator is at least $(\lambda-1) x_{n}$. This gives $1 /(\lambda \mu)$ times the constant in the statement of Property 7. The latter results from consideration of the case in which $(r, s)$ are left-majorized. Here it is required that

$$
C \geqq 4(\alpha \beta)^{2} \frac{\max \left(d_{n}, d_{n+1}\right)}{\min \left(d_{n}, d_{n-1}\right)}
$$

and this produces the extra factor $\lambda \mu$.

To prove the second part assume again that $r$ and $s$ are rightmajorized. Since $r\left(x_{n}\right) \leqq \alpha r\left(x_{n+1}\right)$ and $s\left(x_{n}\right) \leqq \beta s\left(x_{n+1}\right)$ in (17) it suffices now to majorize

by

$$
\alpha \beta \max \left(d_{n}, d_{n+1}\right)(1+\alpha)(1+\beta) r\left(x_{n+1}\right) s\left(x_{n+1}\right)
$$

$$
C \int_{x_{n+1}}^{x_{n+2}} r s d x \geqq \frac{C}{\alpha \beta} d_{n+2} r\left(x_{n+1}\right) s\left(x_{n+1}\right) .
$$

Similar considerations apply when $(r, s)$ are left-majorized. The two together lead to

$$
C \geqq 4(\alpha \beta)^{3} \frac{\max \left(d_{n}, d_{n+1}\right)}{d_{n+2}}, \quad C \geqq 4(\alpha \beta)^{3} \frac{\max \left(d_{n}, d_{n+1}\right)}{d_{n-1}}
$$

when we replace $(1+\alpha)(1+\beta)$ by $4 \alpha \beta$. The second condition leads to the constant in the second inequality of Property 7 .

Property 8. Let $r(x)$ be constant on each interval $\left(x_{n}, x_{n+1}\right)$. Let $s(x)$ be both right-majorized and left-majorized with constant $\alpha$. Then 


$$
\{r \varphi, s \psi\}_{p} \leqq 2 \alpha^{2} \frac{\lambda \mu-1}{\lambda-1} J_{p}(\varphi) J_{q}(\psi)\|r s\|_{1} .
$$

Without loss of generality let $J_{p}(\varphi)=J_{q}(\psi)=1$. To show the structure of the proof more clearly we assume that $s(x)$ is leftmajorized with constant $\beta$ rather than $\alpha$. Then, as in several cases above, it is found that the general term of $\{r \varphi, s \psi\}_{p}$ does not exceed

$$
\left[d_{n+1}^{1 / p} r_{n}+d_{n}^{1 / p} r_{n-1}\right]\left[\beta d_{n+1}^{1 / q}+\alpha d_{n}^{1 / q}\right] s\left(x_{n}\right) .
$$

Here $r_{n}$ is the value of $r$ on $\left(x_{n}, x_{n+1}\right)$. Furthermore, if $C$ is any positive constant,

$$
C \int_{x_{n-1}}^{x_{n+1}} r s d x \geqq C\left[d_{n+1} \frac{r_{n}}{\alpha}+d_{n} \frac{r_{n-1}}{\beta}\right] s\left(x_{n}\right) .
$$

We have to choose $C$ so that the coefficients of $r_{n}$ and $r_{n-1}$ in the latter expression are not smaller than those in the former. This holds if

$$
C \geqq \alpha \beta+\alpha^{2}\left(\frac{d_{n}}{d_{n+1}}\right)^{1 / q}, \quad C \geqq \alpha \beta+\beta^{2}\left(\frac{d_{n+1}}{d_{n}}\right)^{1 / q} .
$$

Thus $C$ must be the larger of the two expressions

$$
\alpha \beta+\alpha^{2}\left(\frac{\mu-1}{\lambda-1} \frac{1}{\mu}\right)^{1 / q}, \quad \alpha \beta+\beta^{2}\left(\frac{\mu-1}{\lambda-1} \lambda\right)^{1 / q} .
$$

The result of Property 8 is obtained by setting $\beta=\alpha$ and considering the worst case, $q=1$. The factor 2 is needed because the intervals $\left(x_{n}, x_{n+1}\right)$ are covered twice in the summation.

9. Inequalities for the weighted norm. Corollary 1 asserts that $\hat{f}_{1} \in L$ provided $f$ admits a certain type of majorant $F \in L$. The proof actually gives $\left\|\hat{f}_{1}\right\|_{1} \leqq C\|F\|_{1}$ where $C$ is some constant, and a similar refinement applies to the other corollaries above. Here we propose to study the dependence of these constants on the other parameters $p, m, \lambda, \mu, \cdots$. The results obtained go farther than previous results in this direction, yet follow with ease from Theorem 1.

COROLlaRY 6. Let $\alpha=a-1 / q$ satisfy $|\alpha|<m$. Then there exists an absolute constant $C$ such that

$$
\left\|x^{a} \hat{f}_{m}\right\|_{p} \leqq C\left(R_{p}+\frac{m}{m-|\alpha|}\right)\left\|x^{a} f\right\|_{p} .
$$

For proof let $a+b=1$ and let $g$ be arbitrary. Then

$$
\{f, x g\}_{p}=\left\{f, x^{a+b} g\right\}_{p} \leqq \mu^{2|a|}\left\{x^{a} f, x^{b} g\right\}_{p}
$$


where in the last step we used Property 5 with $r=x^{a}, s=x^{-a}$. Since $|a|<m+1$ we get, by (15),

$$
\{f, x g\}_{p} \leqq 2 \mu^{2 m+2}\left\|x^{a} f\right\|_{p}\left\|x^{b} g\right\|_{q} .
$$

Here $f=g=0$ for $x<0$. A similar result can be obtained for $x<0$, taking $f=g=0$ for $x>0$. Property 1 gives also

$$
[f, g]_{m} \leqq \frac{8}{m-|\alpha|}\left\|x^{a} f\right\|_{p}\left\|x^{b} g\right\|_{q}
$$

and it remains only to apply Theorem 1.

The exponential dependence on $m$ in $\mu^{m}$ seems a disaster for the estimates desired here, but can be made harmless by appropriate choice of $\mu$. Indeed, the second term in $B_{m}$ does not exceed $m \mu^{2 m}$, and the choice

$$
\lambda=\mu=1+\frac{1}{2 m}
$$

gives $B_{m} \leqq 3 m$. Thus Theorem 1, (19) and (18) together give

$$
\left\|x \hat{f}_{m} g\right\|_{1} \leqq C\left(R_{p}+\frac{m}{m-|\alpha|}\right)\left\|x^{a} f\right\|_{p}\left\|x^{b} g\right\|_{q}
$$

where $C$ is an absolute constant. If we set $G=x^{b} g$ then the left side is $\left\|x^{a} \hat{f}_{m} G\right\|_{1}$, and Corollary 6 follows from the converse to the Schwarz-Hölder inequality. It should be stressed that $C$ is independent of $a, m, p$; the result with $C=C(a, m, p)$ is more immediate.

It is not difficult to show that the constant of Corollary 6 has an appropriate form, in that any such constant must tend to $\infty$ for $p \rightarrow 1$ and $p \rightarrow \infty$ in the same way as does $R_{p}$, and must also tend to $\infty$ as $|\alpha| \rightarrow m$ like $(m-|\alpha|)^{-1}$. Indeed, let $A_{m}, B_{m}$ and $C_{m}$ be any constants such that

$$
\left\|x^{a} \hat{f}_{m}\right\|_{p} \leqq\left(A_{m} R_{p}+\frac{B_{m}}{m-\alpha}+\frac{C_{m}}{m+\alpha}\right)\left\|x^{a} f\right\|_{p}
$$

holds for $|\alpha|<m$ and $1<p<\infty$, as in Corollary 6. Then necessarily

$$
A_{m} \geqq 1, \quad B_{m} \geqq 1, \quad C_{m} \geqq 1, \quad(m=1,2,3, \cdots) .
$$

For proof, let $\delta$ be a small positive constant and let

$$
a=\frac{1}{q}+m-\delta, \quad b=-1-m+\delta+\delta^{2} .
$$

Then the function $f(x)=x^{b}, 0<x<1 ; f(x)=0$ elsewhere, satisfies

$$
\left\|x^{a} f(x)\right\|_{p}<\infty, \quad\left|\hat{f}_{m+1}-\hat{f}_{m}\right| \geqq \frac{f^{\prime}(x)}{\delta+\delta^{2}} .
$$


Since $\left\|x^{a} \hat{f}_{m+1}\right\|_{p}$ is bounded for small $\delta$ by Corollary 6 , letting $\delta \rightarrow 0$ shows that $B_{m} \geqq 1$. Similarly $C_{m} \geqq 1$, and the fact that $A_{m} \geqq 1$ follows from the ordinary Riesz theorem for $a=0$. We do not know whether the numerator $m$ in $m /(m-|\alpha|)$ in Corollary 6 can be replaced by 1. Apart from this, the above example shows that the result is sharp.

The technique of considering $\hat{f}_{m+1}-\hat{f}_{m}$ is a powerful method of constructing examples, and shows that the constants in the following corollaries are also, in various respects, sharp. (Cf. the discussion following Corollary 3.)

CoRollary 7. Let $R_{\delta}$ be the region of the $(\xi, \eta)$ plane defined by

$$
\xi \leqq \frac{1}{q}+m-\delta, \quad \eta \geqq \xi, \quad \eta \geqq \frac{1}{q}-m+\delta
$$

where $\delta>0$. If $(\alpha, \beta)$ and $(\rho, \sigma)$ are two points of $R_{\delta}$, let

$$
\begin{aligned}
& w(x)=|x|^{\beta}, \quad W(x)=|x|^{\alpha}, \quad|x| \leqq 1 ; \\
& w(x)=|x|^{\rho}, \quad W(x)=|x|^{\sigma}, \quad|x| \geqq 1 .
\end{aligned}
$$

Then there exists an absolute constant $C$ such that

$$
\left\|w \widehat{f}_{m}\right\|_{p} \leqq C\left(R_{p}+\frac{m}{\delta}\right)\|W f\|_{p} .
$$

When $\alpha=\beta=\rho=\sigma$ this reduces to Corollary 6 .

For proof, we can assume $\delta \leqq m$. This is so because $R_{p} \geqq \pi$, and hence the term $m / \delta$ can be incorporated with $R_{p}$ by increasing $C$, if $m \leqq \delta$. Also it suffices to establish the result with $(\alpha, \beta)$ and $(\rho, \sigma)$ on the line $\xi=\eta$. This is seen by considerations similar to those used in the proof of Property 2. Thus we can assume that $|\alpha|,|\beta|,|\rho|,|\sigma|$ are at most $m+1$.

We use Property 2 with $r=W, s=x / w$, and with

$$
a=\alpha, \quad b=1-\beta, \quad A=\sigma, \quad B=1-\rho .
$$

It is easily checked that Property 2 applies with the same $\delta$ as in Corollary 7. Hence

$$
[f, g]_{m} \leqq \frac{C_{0}}{\delta}\|r f\|_{p}\|s g\|_{q}
$$

where $C_{0}$ is an absolute constant. In order to estimate $\{f, x g\}_{p}$, consider first the terms for which $x_{n-1} \geqq 1$. In this case $A+B \geqq 1$ gives

$$
\{f, x g\}_{p} \leqq\left\{f, x^{A+B}\right\}_{p} \leqq \mu^{2|A|}\left\{x^{A} f, x^{B} g\right\}_{p}
$$

where in the second step we used Property 5 with $r(x)=x^{4}$. A 
similar result applies to the terms with $x_{n+1} \leqq 1$ and to the single term with $x_{n}=1$. Since $|A| \leqq m+1$ and $|a| \leqq m+1$ can be assumed, as noted above, we get, essentially,

$$
\{f, x g\}_{p} \leqq \mu^{2 m+2}\|r f\|_{p}\|s g\|_{q} \cdot
$$

The result is readily extended to negative $x$. With $\lambda=\mu=1+1 / m$, Theorem 1 gives an absolute constant $C$ such that

$$
\left\|\hat{f}_{m} x g\right\|_{1} \leqq C\left(R_{p}+\frac{m}{\delta}\right)\|r f\|_{p}\|s g\|_{q} .
$$

(A similar inequality can be found with more general $r$ and $s$ by using (14) together with the full force of Property 5.) As before, we set $G=x g$ and use the converse to the Schwarz-Hölder inequality. The result is Corollary 7 .

The discussion following Corollary 6 indicates that some of the inequalities in the hypothesis of Corollary 7 are appropriate, but gives no clue concerning the inequality $\eta \geqq \xi$. This is discussed now, under the hypothesis that the remaining conditions

$$
\xi-\frac{1}{q}<m, \quad \eta-\frac{1}{q}>-m,
$$

continue to hold. We shall show that the conclusion of Corollary 8 is never true if $\alpha>\beta$, or if $\rho>\sigma$; in other words, the condition $\eta \geqq \xi$ is really needed both times it is used.

To this end, note that $\hat{f}_{m}$ can be written in the form

$$
\hat{f}_{m}(x)=\frac{1}{x} \int_{-\infty}^{\infty} L_{m}\left(\frac{u}{x}\right) f(u) d u .
$$

If $f(x)=x^{a}$ for $0<x<1$, and $f(x)=0$ otherwise, the substitution $u=t x$ gives

$$
\hat{f}_{m}(x)=x^{2} \int_{0}^{1 / x} L_{m}(t) t^{a} d t, \quad x>0,
$$

and so $\hat{f}_{m}(x) \sim c_{a} x^{a}$ as $x \rightarrow 0+$ where $c_{a}$ is a certain definite integral. This integral converges if $|a+1|<m$. For present purposes we take a in such a way that

$$
\frac{1}{q}-\xi<a+1<\frac{1}{q}-\eta
$$

which is always possible if $\xi>\eta$. The above conditions on $\xi$ and $\eta$ ensure the needed inequality $|a+1|<m$. The inequality

$$
\int_{0}^{1}|x|^{\gamma p p}\left|\hat{f}_{m}(x)\right|^{p} d x \leqq C \int_{0}^{1}|x|^{\xi p}|f(x)|^{p} d x+\int_{1}^{\infty} W(x)|f(x)|^{p} d x
$$


fails for this function $f$ no matter what the weight $W$ may be, since the integral on the left diverges while both integrals on the right converge. This shows that $\alpha \leqq \beta$ is necessary in Corollary 7. Similary, $\rho \leqq \sigma$ is necessary.

10. Majorants of special forms. Here the results of Corollaries 1-5 are refined and extended. The reason for presenting Corollaries 1-5 separately is that their proofs are very simple, while the extensions obtained now require more attention to detail. If only the existence of some constant $C$ is required, the considerations of this section can be simplified after the manner of Corollaries 1-5, and the rather tedious analysis of $\S \S 7$ and 8 can be, in part, avoided.

In the minimal decomposition $f=F \phi, F$ could be expressed as a difference of two step functions both of which are monotone. Thus $f$ for $x>0$ can be written as a difference of two expressions $F \varphi$ in which $J_{p}(\varphi) \leqq 1, F$ is constant on each interval $\left(x_{n}, x_{n+1}\right)$, and $F$ is increasing.

Often such a majorant $F$ can be majorized by another in which $x^{-a} F(x)$ is decreasing for some $a$. Thus we are led to consider majorants $F \Phi \geqq|f|$ in which, instead of being a step function, $F$ has the property that $F x^{a}$ increases and $F x^{-a}$ decreases. Similar remarks apply to the weight $g$; that is, we consider $|g| \leqq G \psi$ where $J_{q}(\psi) \leqq 1$ and where $G$ has various regularity properties. As noted above, the $L^{1}$ behavior of $\hat{f}_{m}$ relative to the weight $g$ is largely determined by the $L^{1}$ behavior of $F G$ in these decompositions.

In this section it is assumed that

$$
|f(x)| \leqq F(|x|) \varphi(|x|), \quad|g(x)| \leqq G(|x|) \psi(|x|)
$$

for $-\infty<x<\infty$, where

$$
F(x)=G(x)=0 \text { for } x<0, \quad F(x)>0, \quad G(x)>0 \text { for } x>0
$$

and where, relative to $\left\{x_{n}\right\}$,

$$
J_{p}(\varphi) \leqq 1, \quad J_{q}(\psi) \leqq 1 .
$$

Corollary 8. Let (20) hold and suppose further:

(i) $F(x)$ is constant on each interval $\left(x_{n}, x_{n+1}\right)$;

(ii) $x^{-a} G(x)$ decreases and $x^{a} G(x)$ increases for some $a<m$. Then there exists a constant $C$, depending on $\mu^{m}$ alone, such that

$$
\left\|\hat{f}_{m} g\right\|_{1} \leqq C \frac{\mu-1}{\lambda-1}\left(R_{p}+\frac{\mu-1}{m-a}\right)\|F G\|_{1} .
$$

Clearly $a \geqq 0$ unless $g=0$, in which case the theorem is vacuous. 
If $\mu^{m}$ is bounded-for example, if $\mu \leqq(1+1 / m)^{2}$-then $C$ is an absolute constant. We can always diminish $\lambda$ so as to make $\lambda \leqq 1+$ $1 / m$, since the inequality $x_{n+1} / x_{n}>\lambda$ continues to hold with the new $\lambda$. We can then add enough geometric means on each interval $\left(x_{n}, x_{n+1}\right)$ so as to make $\mu \leqq \lambda^{2}$ for the new sequence. This operation multiplies $J_{p}(\varphi)$ and $J_{q}(\psi)$ by factors which do not exceed, respectively,

$$
\left(\frac{\mu-1}{\lambda-1}\right)^{1 / p},\left(\frac{\mu-1}{\lambda-1}\right)^{1 / q} \text {. }
$$

Hence, if $\lambda \leqq 1+1 / m$, we can replace $C$ by

$$
C_{0} \frac{\mu-1}{\lambda-1}
$$

where $C_{0}$ is an absolute constant.

In the proof it is convenient to use $C_{k}$ to denote various constants depending on $\mu^{m}$ alone. We also note the relations

$$
J_{1}(\varphi) \leqq J_{p}(\varphi) \leqq 1, \quad J_{1}(\psi) \leqq J_{q}(\psi) \leqq 1
$$

whice follow from the fact that $J_{p}(\varphi)$ is a monotone function of $p$. Furthermore, the assumed monotony of $G$ ensures that $G(x)$ is both right and left majorized with constant $\mu^{a} \leqq \mu^{m}$.

For the first step of the proof, Property 4 gives

$$
[f, g / x]_{m} \leqq 2[G \psi / x, f]_{m} \leqq C_{1}[G / x, f]_{m} J_{1}(\psi) \leqq C_{1}[G / x, f]_{m} .
$$

For the second step, Property 2 gives

$$
[f, G / x]_{m} \leqq 2[F \varphi, G / x]_{m} \leqq \frac{4}{m-a}\|F \varphi G\|_{1} .
$$

For the third step, since $G$ is right-majorized with constant $\mu^{m}$,

$$
\int_{x_{n}}^{x_{n+1}}|F \varphi G| d x \leqq \mu^{m} F\left(x_{n}\right) G\left(x_{n+1}\right)\left(x_{n+1}-x_{n}\right)
$$

where again we have used $J_{1}(\varphi) \leqq J_{p}(\varphi) \leqq 1$. On the other hand since $G$ is left-majorized with constant $\mu^{m}$

$$
\int_{x_{n}}^{x_{n+1}} F G d x \geqq \mu^{-m} F\left(x_{n}\right) G\left(x_{n+1}\right)\left(x_{n+1}-x_{n}\right) .
$$

Hence by addition $\|F \varphi G\|_{1} \leqq C_{2}\|F G\|_{1}$. These three steps together give

$$
[f, g]_{m} \leqq \frac{C_{3}}{m-a}\|F G\|_{1}
$$

For the fourth step, Property 8 gives 


$$
\{f, g\}_{p} \leqq C_{4} \frac{\mu-1}{\lambda-1}\|F G\|_{1} .
$$

Since $B_{m}$ in Theorem 1 clearly satisfies

$$
B_{m} \leqq \frac{C_{5}}{\lambda-1}
$$

Theorem 1 gives the desired conclusion in case $g(x)=0$ for $x<0$.

To get the full result, note that the two relations

$$
\hat{f}_{m}(x)=\frac{1}{x} \int_{-\infty}^{\infty} L_{m}\left(\frac{u}{x}\right) f(u) d u, \quad \hat{f}_{m}(-x)=-\frac{1}{x} \int_{-\infty}^{\infty} L_{m}\left(\frac{u}{x}\right) f(-u) d u
$$

imply each other. In other words, $\hat{f}_{m}(-x)$ is the modified Hilbert transform of $-f(-u)$. Since

$$
\int_{-\infty}^{0}\left|g(x) f_{m}(x)\right| d x=\int_{0}^{\infty}\left|g(-x) f_{m}(-x)\right| d x
$$

and since $g(-x)$ and $f(-u)$ satisfy the same hypothesis as $g(x)$ and $f(u)$, the above estimate applies to the integral (23). Addition of the two estimates gives Corollary 8.

It has already been mentioned that the assumption $|f| \leqq F \varphi$, or even $|f|=F \varphi$, with $F$ and $\varphi$ as in Corollary 8 , holds for every $f$ which is locally $L^{p}$. Sometimes $|f| \leqq H \varphi$ where $H(x)$ is right or left majorized with some constant $\alpha$. In this case we can obtain a majorant $F(x)$ by taking $F(x)=\alpha H\left(x_{n}\right)$ or $\alpha H\left(x_{n+1}\right)$ on $\left(x_{n}, x_{n+1}\right)$ and can apply Corollary 8 (cf. in this connection the deduction of Corollary 2 from Corollary 1). In particular, Corollary 8 applies if $x^{\circ} H(x)$ is monotone for some $\rho$.

When $|f|$ has such a majorant, it is often possible to get by with only a one-sided monotony condition on $G$. We shall establish:

CoRollary 9. Let (20) hold and suppose there exists a constant $a<m$ such that one of the following holds:

(i ) $x^{1-a} F(x)$ and $x^{-a} G(x)$ both decrease, or

(ii) $x^{1+a} F(x)$ and $x^{a} G(x)$ both increase.

Then there exists a constant $C$, depending on $\mu^{m}$ alone, such that

$$
\left\|\hat{f}_{m} g\right\|_{1} \leqq C \frac{\mu-1}{\lambda-1}\left(R_{p}+\frac{\mu-1}{(\lambda-1)^{2}} \frac{1}{m-a}\right)\|F G\|_{1} .
$$

The proof is similar to the above proof. Two uses of Property 4 followed by one use of Property 2 give

$$
[F \varphi, G \dot{\gamma} / x]_{m} \leqq \frac{C_{1}}{m-a} \frac{(\mu-1)^{2}}{(\lambda-1)^{2}}\|F G\|_{1} .
$$


On the other hand Property 7 gives

$$
\{F \varnothing, G \psi\}_{p} \leqq C_{2} \frac{\mu-1}{\lambda-1}\|F G\|_{1} .
$$

Corollary 9 now follows from Theorem 1.

In Corollary 8 the roles of $f$ and $g$ could be reversed, giving a corresponding inequality in which $f$ is associated with a monotone majorant and $g$ is associated with a function $G$ constant on each interval. The new result applies when $G$ is right or left majorized with some constant $b$, and is stated as follows:

CoRollary 10. Let (20) hold and suppose further:

(i) $x^{1-a} F(x)$ decreases and $x^{1+a} F(x)$ increases for some constant $a<m$;

(ii) $G(x)$ is right-or left-majorized with some constant b. Then there exists a constant $C$, depending on $\mu^{m}$ alone, such that

$$
\left\|\hat{f}_{m} g\right\|_{1} \leqq C \frac{\mu-1}{\lambda-1}\left(b^{3} R_{p}+\frac{b^{2}}{(\lambda-1)(m-a)}\right)\|F G\|_{1} .
$$

Here Property 4 used twice followed by Property 2 gives

$$
[F \varphi, G \psi / x]_{m} \leqq C_{1} b^{2} \frac{\mu-1}{\lambda-1} \frac{1}{m-a}\|F G\|_{1} .
$$

Property 7 gives

$$
\{F \varphi, G \psi\}_{p} \leqq C_{2} b^{3} \frac{\mu-1}{\lambda-1}\|F G\|_{1}
$$

and again the conclusion follows from Theorem 1 . The result applies with $b=\mu^{|\rho|}$ if $x^{\rho} G(x)$ is monotone for some $\rho$. The roles of $f$ and $g$ can be interchanged, giving Corollary 8 again for the case in which $F$, instead of being constant, is right or left majorized with some constant $b$.

11. Concluding remarks. It should perhaps be mentioned again that an even majorant as in (20) is not really necessary. If, instead, we refer $f(-x)$ and $g(-x)$ to another sequence $\left\{y_{n}\right\}$ belonging to $(\lambda, \mu)$, the most important change is that $\|F G\|_{1}$ must now be replaced by

$$
\|F(x) G(x)\|_{1}+\|F(-x) G(x)\|_{1} .
$$

With a more fussy arrangement of details, it is found that an estimate for $x>0$ involves the special majorants $F, G$ only for $x>0$, just as $\{f, g\}_{p}$ is required only for $x>0$. Thus we get inequalities of the general form 


$$
\int_{0}^{\infty}\left|\hat{f}_{m}(x) g(x)\right| d x \leqq C_{1} \int_{0}^{\infty} F(x) G(x) d x+C_{2} \int_{0}^{\infty}|f(-x) g(x)| d x .
$$

As a natural continuation of this sequence of corollaries, one might consider the case in which both $F$ and $G$ are constant on the intervals $\left(x_{n}, x_{n+1}\right)$, so that $F \varphi$ and $G \psi$ are both minimal decompositions. This can be done, but the resulting statement is unappealing because of the overlapping in $\{f, g\}_{p}$. That is, values of $F$ on $\left(x_{n}, x_{n+1}\right)$ are correlated with the values of $G$ on $\left(x_{n+1}, x_{n+2}\right)$, as well as with those on $\left(x_{n}, x_{n+1}\right)$, and no hypothesis on $\|F G\|_{1}$ alone can ensure convergence of the series. In the special case in which $\left\{x_{n}\right\}$ is a geometric sequence, so that $\lambda=\mu$, the integrals encountered are essentially of the form

$$
\|F(x) G(x)\|_{1}, \quad\|F(x) G(\lambda x)\|_{1} .
$$

Formulation of the theorem in this case is left to the reader.

For suitably restricted classes these conditions may be both necessary and sufficient, in the following sense: If $\|F G\|_{1}=\infty$, one can construct functions $f=F \varphi, g=G \psi$, with $J_{p}(\varphi) \leqq 1, J_{q}(\psi) \leqq 1$, and such that $\left\|\hat{f}_{m} g\right\|_{1}=\infty$. This seems tolerably clear from the proof of Theorem 1, but detailed investigation is reserved for another occasion. The question of necessary and sufficient conditions is taken up from a different point of view in [2], [9], [13] and [17], but none of these results apply to the $L^{1}$ case of interest here.

Another question to which the solution is not presently known is this: For precisely what classes of function-pairs $(f, g)$ do the two conditions

$$
\left\|x \widehat{f}_{m} g\right\|_{1}<\infty, \quad\left\|x f \hat{g}_{m}\right\|_{1}<\infty
$$

imply each other? A third question is this: To what extent do the results hold if, instead of belonging to $(\lambda, \mu)$, the sequence $\left\{x_{n}\right\}$ satisfies $\sum\left(y_{n} / x_{n}\right)^{2}=\infty$ only, where $y_{n}=x_{n+1}-x_{n}$ ?

\section{REFERENCES}

1. K. I. Babenko, On conjugate functions, Dok. Akad. Nauk SSR (N.S.), 62 (1948), 157-160.

2. Ralph P. Boas, Jr., Integrability Theorems for Trigonometric Transforms, Springer 1967.

3. A. P. Calderón and A. Zygmund, On the existence of certain singular integrals, Acta Math., 88 (1952), 85-139.

4. K. Chen, On absolute Cesàro summability of negative order for a Fourier series at a given point, Amer. J. Math., 66 (1944), 299-312.

5. Yung-Ming Chen, Theorems of asymptotic approximation, Math. Ann., $\mathbb{1 4 0}$ (1960), 360-407.

6. - On conjugate functions, Canadian J. Math. 15 (1963), 486-494.

7. Mischa Cotlar, Condiciones de continuidad de operadores potenciales y de Hilbert, 
Cursos y seminarios de mathematica, 2, Univ. de Buenos Aires, (1959), 210-211.

8. T. M. Flett, Some theorems on odd and even functions, Proc. London Math. Soc., (3) 8 (1958), 135-148.

9. V. F. Gaposkin, A generalization of M. Riesz's theorem on conjugate functions, M. Sbornik (88), 46 (1958), 359-372.

10. G. H. Hardy and J. E. Littlewood, Some more theorems concerning Fourier series and Fourier power series, Duke Math. J., 2 (1936), 354-382.

11. G. H. Hardy, J. E. Littlewood and G. Pólya, Inequalities, Cambridge University Press, (1959), p. 229.

12. G. H. Hardy, J. E. Littlewood and G. Pólya, Inequalities, Cambridge University Press, (1959), p. 246.

13. Henry Helson and G. Szegö, Problem in prediction theory, Ann. di Math. Bologna IV, 51 (1960), 107-138

14. H. Ishikawa and Sumiyuki Koizumi, On some theorems of the Fourier transform, J. Fac. Sci. Hokkaido Univ., Sapporo 14 (1958), 225-230.

15. Sumiyuki Koizumi, On the Hilbert transform I, II, J. Fac. Sci. Hokkaido Univ., Sapporo, 14 (1959), 153-224; 15 (1960), 93-130.

16. - On the singular integrals, V, Proc. Japan. Acad. Tokyo, 35 (1959), 1-6.

17. Paul Koosis, Weighted quadratic means of Hilbert transforms, to appear.

18. R. M. Redheffer, An inequality for the Hilbert transform, Proc. Nat. Acad. Sci. 61 (1968), 810-811.

19. - On a theorem of V. I. Matzayev, Duke Math. J., to appear.

20. A. Zygmund, On a theorem of Marcinkiewicz concerning interpolation of operations, J. de Math., 35 (1956), 223-248.

Received July 26, 1967, and in revised form September 11, 1970. The preparation of this paper was supported in part by NSF Grant $\#$ GP-13377.

University of CALifornia, Los ANGeles 



\section{PACIFIC JOURNAL OF MATHEMATICS}

\section{EDITORS}

H. SAMELSON

Stanford University

Stanford, California 94305

C. R. Новву

University of Washington

Seattle, Washington 98105
J. DUGundJI

Department of Mathematics

University of Southern California

Los Angeles, California 90007

RICHARD ARENS

University of California

Los Angeles, California 90024

\section{ASSOCIATE EDITORS}
E. F. BECKENBACH
B. H. NeumanN
F. WOLF
K. YOSHIDA

\section{SUPPORTING INSTITUTIONS}
UNIVERSITY OF BRITISH COLUMBIA
CALIFORNIA INSTITUTE OF TECHNOLOGY
UNIVERSITY OF CALIFORNIA
MONTANA STATE UNIVERSITY
UNIVERSITY OF NEVADA
NEW MEXICO STATE UNIVERSITY
OREGON STATE UNIVERSITY
UNIVERSITY OF OREGON
OSAKA UNIVERSITY
UNIVERSITY OF SOUTHERN CALIFORNIA
STANFORD UNIVERSITY
UNIVERSITY OF TOKYO
UNIVERSITY OF UTAH
WASHINGTON STATE UNIVERSITY
UNIVERSITY OF WASHINGTON
AMERICAN MATHEMATICAL SOCIETY CHEVRON RESEARCH CORPORATION TRW SYSTEMS
NAVAL WEAPONS CENTER 


\section{Pacific Journal of Mathematics}

\section{Vol. 37, No. $1 \quad$ January, 1971}

Gregory Frank Bachelis and Haskell Paul Rosenthal, On unconditionally

converging series and biorthogonal systems in a Banach space .........

Richard William Beals, On spectral theory and scattering for elliptic

operators with singular potentials .........................

J. Lennart (John) Berggren, Solvable and supersolvable groups in which every element is conjugate to its inverse ........................ 21

Lindsay Nathan Childs, On covering spaces and Galois extensions ..........

William Jay Davis, David William Dean and Ivan Singer, Multipliers and

unconditional convergence of biorthogonal expansions..............

Leroy John Derr, Triangular matrices with the isoclinal property ............

Paul Erdős, Robert James McEliece and Herbert Taylor, Ramsey bounds for

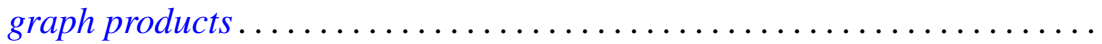

Edward Graham Evans, Jr., On epimorphisms to finitely generated

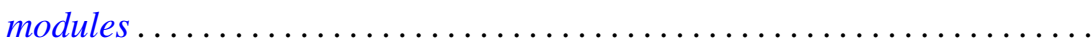

Hector O. Fattorini, The abstract Goursat problem ................. 51

Robert Dutton Fray and David Paul Roselle, Weighted lattice paths .........

Thomas L. Goulding and Augusto H. Ortiz, Structure of semiprime $(p, q)$

radicals ...........................................

E. W. Johnson and J. P. Lediaev, Structure of Noether lattices with join-principal maximal elements ....

David Samuel Kinderlehrer, The regularity of minimal surfaces defined over

slit domains

Alistair H. Lachlan, The transcendental rank of a theory. .

Frank David Lesley, Differentiability of minimal surfaces at the boundary ...

Wolfgang Liebert, Characterization of the endomorphism rings of divisible torsion modules and reduced complete torsion-free modules over complete discrete valuation rings....

Lawrence Carlton Moore, Strictly increasing Riesz norms.

Raymond Moos Redheffer, An inequality for the Hilbert transform ...

James Ted Rogers Jr., Mapping solenoids onto strongly self-entwined,

circle-like continua..........................

Sherman K. Stein, B-sets and planar maps ................... 217

Darrell R. Turnidge, Torsion theories and rings of quotients of Morita

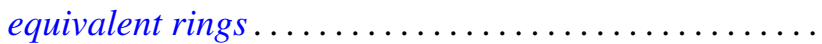

Fred Ustina, The Hausdorff means of double Fourier series and the principle of localization ................................

Stanley Joseph Wertheimer, Quasi-compactness and decompositions for arbitrary relations.

Howard Henry Wicke and John Mays Worrell Jr., On the open continuous images of paracompact $\check{C}$ ech complete spaces... 